\title{
Estudo do membro pélvico do avestruz: considerações anatômicas, radiográficas e histológicas da rotação tibiotársica
}

\author{
Study of hind limb in ostrich: anatomical, radiographic and histological considerations \\ of tibiotarsal rotation
}

\section{Roselaine Ponso de OLIVEIRA ${ }^{1}$; Gisele SAVIANI ${ }^{1}$; Haley Silva de CARVALHO ${ }^{1}$; Vanessa Belentani MARQUES $^{1}$; Ricardo de ALBUQUERQUE ${ }^{2}$}

${ }^{1}$ Departamento de Cirurgia da Faculdade de Medicina Veterinária e Zootecnia da Universidade de São Paulo, São Paulo - SP

${ }^{2}$ Departamento de Nutrição e Produção Animal da Faculdade de Medicina Veterinária e Zootecnia da Universidade de São Paulo, Pirassununga - SP

\begin{abstract}
Resumo
Várias doenças músculo esqueléticas foram observadas em aves de rápido crescimento, sendo o elevado peso corporal, associado a uma estrutura óssea em formação, citados como as principais causas responsáveis pela ocorrência destas doenças, resultando em disfunções do aparelho locomotor. O presente trabalho teve como objetivo fazer um estudo anátomo-patológico e radiográfico das estruturas envolvidas na rotação tibiotársica, assim como estudar as alterações articulares por meio de artrografias e histologia fazendo uma descrição das considerações anatômicas, macroscópicas, microscópicas e radiográficas do membro pélvico do avestruz (African Black). Dessa forma, será possível contribuir para o entendimento desta enfermidade, que tem causado perdas para a estrutiocultura.
\end{abstract}

Palavras-chave: Anatomia animal. Radiologia animal. Histologia animal. Avestruz. Tibiotársica.

\begin{abstract}
Many skeletal muscle diseases were observed in fast growth birds, and the high body weight, associated to a bone structure in formation are named to be the principal responsible causes for the occurrence of these diseases, which results in dysfunction of the locomotor apparatus. This present research intends to make an anatomical, pathological and radiographic study of the structures involved in the tibiotarsal rotation, besides the study of the joint alterations by arthrography and histology, giving a description with anatomical, macroscopic, microscopic and radiographic considerations of the ostrich's hind limbs (African Black). This way, it'll be possible to contribute to the comprehension of this disease that has caused many losses on the ostrich farm.
\end{abstract}

Keywords: Animal anatomy. Animal radiology. Animal histhology. Ostrich. Tibiotarsal.

\section{Introdução}

O avestruz (Struthio camelus) é uma espécie extremamente antiga e tem adquirido importância econômica nos últimos anos pela diversidade de produtos no mercado interno originados de sua criação.

Sua criação iniciou-se na África do Sul em torno de 1863, com a captura de aves selvagens. Sofreu um processo de rigorosa seleção para a produção de plumas de qualidade e carne com baixo teor de colesterol desde o início do século $\mathrm{XX}^{1}$.

"Anormalidades de pernas, pernas tortas ou curvas, ou problemas de pernas", são termos comumente en- contrados na literatura, usados para descrever condições que envolvam deformidades nos ossos, articulações, com ou sem anormalidades de cartilagem, ou desvio do tendão gastrocnêmio.

Fatores nutricionais, doenças genéticas e fatores ligados ao meio ambiente, o rápido ganho de peso de-

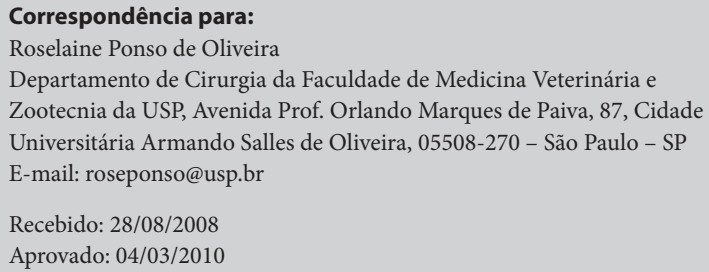


vido a dietas energéticas e hiperproteicas, frequentemente contribuem para produção de um problema de desenvolvimento específico.

Foram estes fatores que despertaram a atenção para o estudo que objetiva a descrição das particularidades morfológicas observadas na rotação tibiotársica, bem como dar suporte para novas pesquisas sobre problemas osteoarticulares nos membros pélvicos das aves de rápido crescimento.

\section{Material e Método}

Para o presente estudo foram selecionados 30 avestruzes, com idades entre um mês a um ano e meio, machos ou fêmeas, provenientes do criatório da Brasil Ostrich, Unesp - Araçatuba e Fazenda Porto Feliz. Realizamos o estudo radiográfico, artrográfico e histológico.

No primeiro lote de avestruzes que foram encaminhados ao exame radiográfico anteriormente ao abate, as radiografias foram realizadas no Hospital Veterinário da Faculdade de Medicina Veterinária e Zootecnia da Universidade de São Paulo - Pirassununga, com a utilização de aparelho portátil, para que os animais tivessem o mínimo de estresse. Foi realizada a contenção mecânica, segundo técnica empregada por Shane e Jully ${ }^{2}$ por meio do uso de capuz, por serem animais destinados a abate e consumo. Os animais foram mantidos em estação.

Os filmes radiográficos utilizados foram MXG/Plus de tamanho $24 \times 30$ centímetros". A revelação e fixação dos filmes foram efetuadas em processadora manual.

No exame radiográfico dos membros as tomadas radiográficas foram realizadas nas posições craniocaudal e mediolateral. Deste lote foi colhido o material para histologia.

O segundo lote de animais utilizados consistia de vinte filhotes de avestruz, e dois avestruzes jovens, congelados, que vieram a óbito por causas desconhecidas em suas propriedades de origem, que foram radiografados no Hospital Veterinário da Faculdade de
Medicina Veterinária e Zootecnia da Universidade de São Paulo - sendo as radiografias realizadas com aparelho fixo.

Os filmes radiográficos utilizados foram MXG/Plus de tamanho $18 \times 24$ centímetros .

Os animais foram descongelados por 24 horas, sendo então realizada a perfusão, cuja via de acesso foi a aorta, com solução fisiológica em temperatura ambiente, fixados e formolizados e feita a injeção de solução de Laskovisk modificado por um período de aproximadamente duas horas. Realizou-se então o estudo radiográfico das articulações intertársica e metatarsofalangeana.

Posteriormente ao estudo radiográfico, foi realizado o estudo contrastado positivo, utilizando-se o iohexol, que é um meio de contraste iodado, hidrossolúvel, monomérico e não iônico, na concentração de $300 \mathrm{mg} / \mathrm{ml}$, com osmolaridade de $672 \mathrm{mmol} / \mathrm{Kg}$, administrado no volume de aproximadamente 2 a 7 $\mathrm{ml}$ conforme o tamanho do animal, nas mesmas projeções radiográficas. Os animais eram posicionados em decúbito lateral e dorsal, com as articulações intertársica, tarsometatarsica-falangeanas e metatársicofalangeana, voltadas para cima, onde aproximadamente a $1 \mathrm{~cm}$ distal e caudal, das cápsulas articulares, era inserida a agulha fina acoplada na seringa de $5 \mathrm{ml}$ até atingir o espaço articular, removendo-se pequeno volume do líquido sinovial, para confirmação da localização. Em seguida, injetava-se o contraste com o volume aproximado ao do líquido sinovial retirado. Promoviam-se movimentos de flexão e extensão das articulações para distribuição do meio de contraste. As radiografias foram efetuadas logo após a aplicação com os mesmos posicionamentos praticados ao exame radiográfico simples. Os filmes foram colocados em chassis metálicos com telas intensificadoras Lanex, e foram revelados em processadora manual.

Após o estudo radiográfico efetuado no primeiro lote de animais, que foram encaminhados ao HOVET de Pirassununga, o mesmo lote foi conduzido ao aba- 
tedouro escola, onde se procedeu a coleta do material para a histologia.

Dos animais abatidos foram colhidas as extremidades proximais do tibiotarso do antímero direito e esquerdo, que após a fixação em formalina a $20 \%$ por quatro dias, foram descalcificadas em ácido fórmico a $10 \%$ tamponado com citrato de sódio, $\mathrm{pH} 4,5$, sob vácuo moderado, por 24 a 48 horas. Foram feitos cortes em fragmentos, que foram desidratados em soluções alcoólicas de concentração crescente, diafanizadas em xilol e incluídos em parafina. Cortes de aproximadamente cinco micra de espessura, mostrando os contornos longitudinais, foram obtidos e corados pelo método da Hematoxilina-Eosina (HE) conforme técnicas usuais.

A análise destes foi feita em microscópio óptico (Leitzbinocular, aumento 100x e 400x) e realizadas fotomicrografias. O processamento do material foi realizado no Laboratório de Histologia do Departamento de Patologia da Faculdade de Medicina Veterinária da Universidade de São Paulo.

\section{Resultados}

As imagens foram selecionadas para melhor ilustrar os achados e foram documentadas por meio de fotografias digitais e desenhos esquemáticos.

O exame radiográfico demonstrou alterações nos eixos ósseos e degeneração articular, confirmando lesões suspeitadas na avaliação clínica dos animais.

Pelas imagens radiográficas notou-se que a rotação ocorre mais freqüentemente na tíbia, sendo menos evidentes em tarsometatarso e falanges. Defeitos de tibiotarso proximal ocorreram com mais frequência nos membros afetados. Linhas transversas espessadas foram frequentemente observadas em tibiotarso e tarsometatarso proximal, nos membros mais deformados. Identificou-se curvatura em diáfise onde estas linhas transversais espessadas foram localizadas na extremidade distal do osso proximal a articulação tibiotársica. Em alguns casos foram encontrados subluxação de articulação metatarsofalangeana.
A seguir são descritas as observações efetuadas nas figuras apresentadas. Na figura 1 - Projeção craniocaudal das articulações intertársicas, observa-se desvio curvo do terço proximal dos tarsometatarsos: nota-se

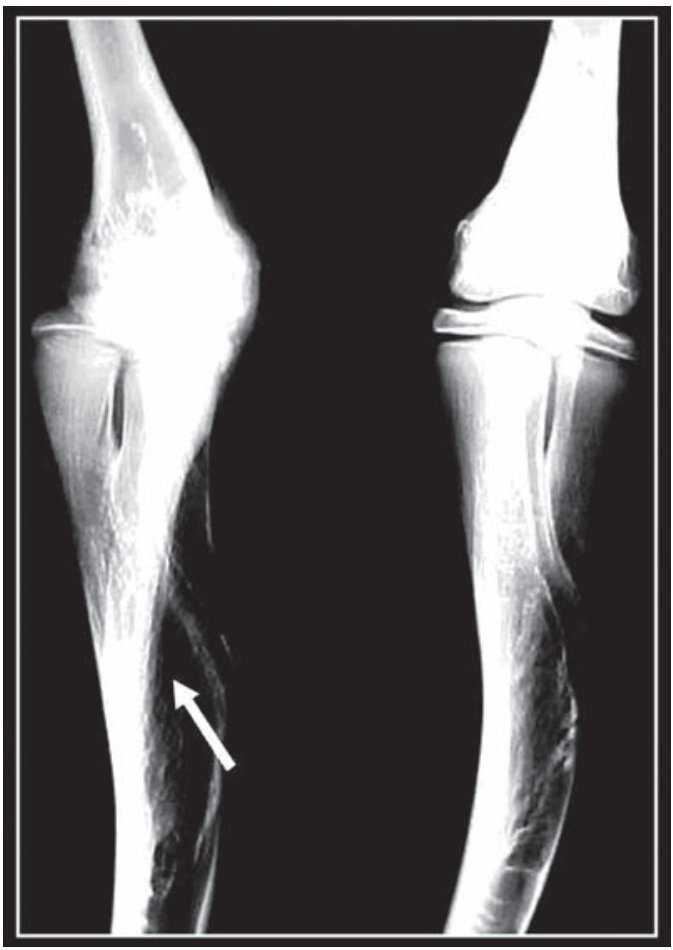

Figura 1 - Projeção craniocaudal das articulações intertársicas, observa-se desvio curvo do terço proximal dos tarsometatarsos: notase espessamento da cortical e linhas de estresse(seta). Idade: 8 meses

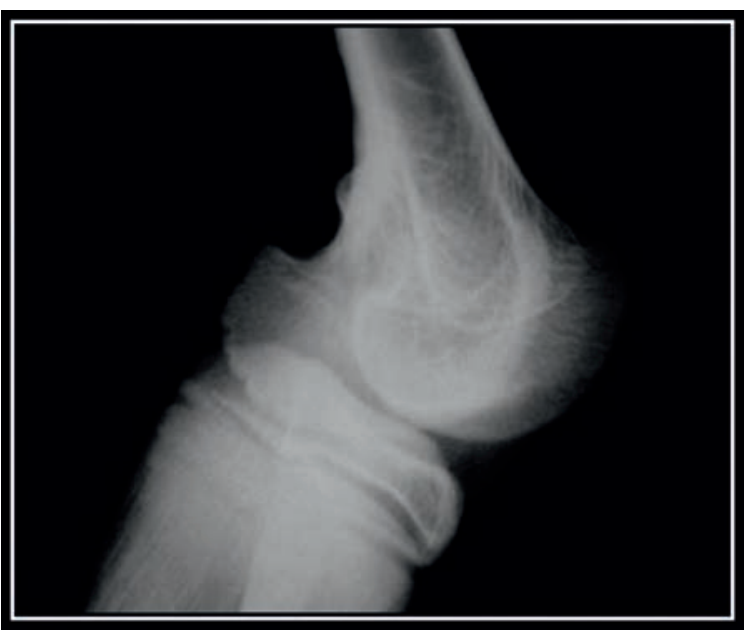

Figura 2 - Projeção médiolateral da articulação intertarsica de um animal jovem, apresentando irregularidade do osso subcondral em côndilos do tíbiotarso (degeneração articular) (seta) 
espessamento da cortical e linhas radiopacas horizontais (linhas de retardo de crescimento). Na figura 2 Projeção mediolateral da articulação intertársica com deformidade do osso subcondral em tarsometatarso e fragmento intra-articular (degeneração articular). As figuras 3 e 4 demonstraram a presença de centro de ossificação secundária com a presença de condrócitos hipertróficos. Observa-se a presença de canal de

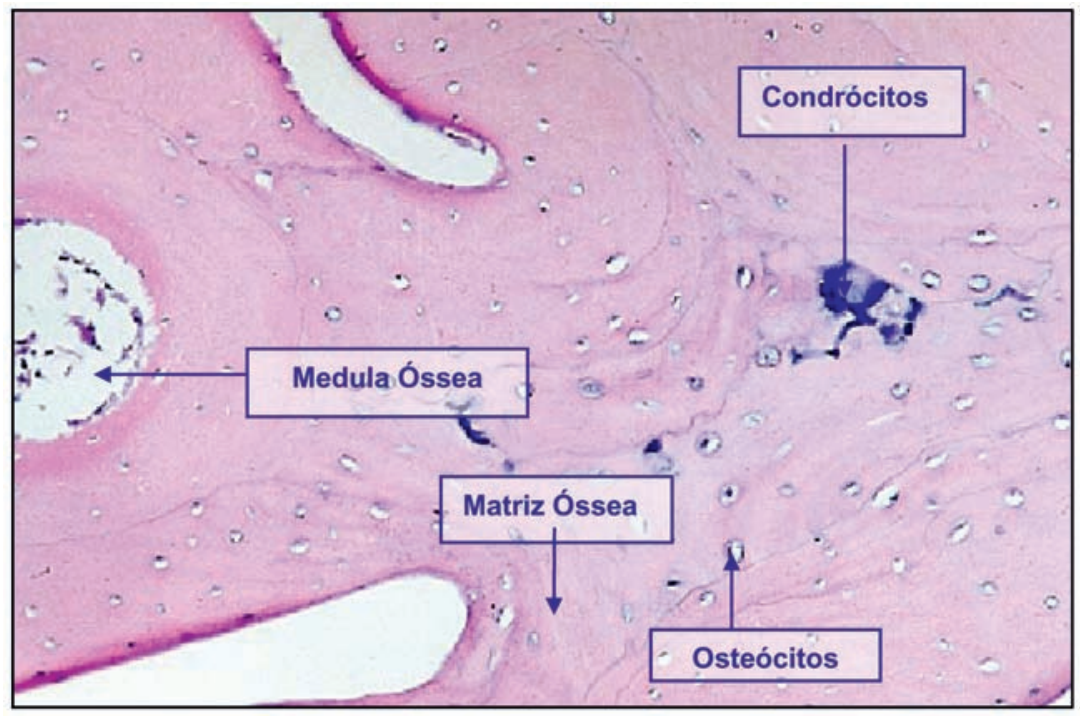

Figura 3 - Fotomicrografia mostrando corte transversal de tíbia de avestruz, descalcificado e corado pela técnica da hematoxilina e eosina onde se pode observar osteócitos e a presença de células cartilaginosas (condrócitos) no interior da matriz óssea. Notar parte da medula óssea. Aumento 110X

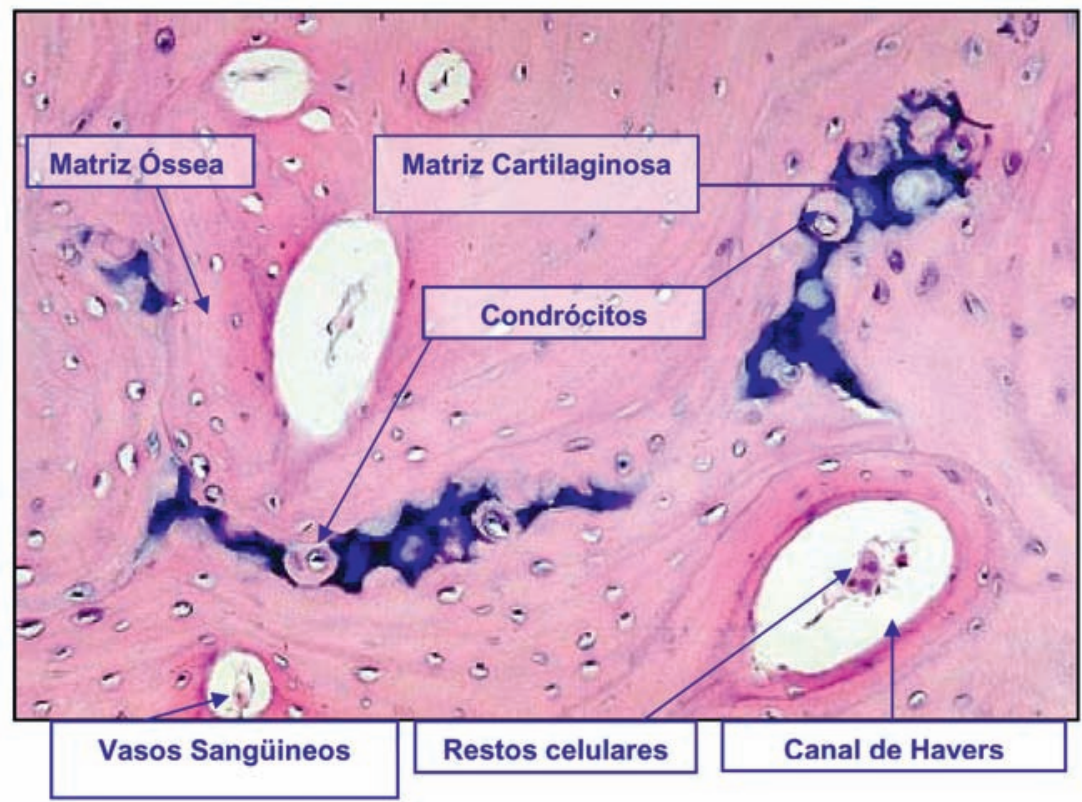

Figura 4 - Fotomicrografia mostrando corte transversal de tíbia de avestruz descalcificado e corado pela técnica da hematoxilina e eosina. Notar a presença de células cartilaginosas (condrócitos) e remanescente de matriz cartilaginosa no interior da matriz óssea. Notar no interior do canal de Havers, a presença de restos celulares. Aumento 110X 
Havers ao corte de osso compacto. Remodelagem óssea zona de transição e osteócitos. Observam-se fibras colágenas dispostas paralelamente com poucas células, caracterizando um tecido conjuntivo denso modelado.

Na porção celular constatamos a presença de vasos sanguíneos com diâmetro diferente e alguns apresentando hemáceas no seu interior.

\section{Discussão}

O propósito do presente estudo foi fazer um estudo anátomo patológico e radiográfico das estruturas envolvidas na rotação tibiotársica observando possíveis alterações macro e microscópicas.

Diversos autores apontam a origem do avestruz na África do Sul ${ }^{1,3,4}$, citando-o como a maior ave do mundo, salientando o interesse em introduzir no mercado os principais produtos originados da estrutiocultura - carne, plumas, pele.

Huchzermeyer ${ }^{1}$ e Speer ${ }^{5}$ concordam que problemas de alterações de crescimento são geralmente encontrados, o que é reforçado por Bezuidenhout et al. $^{6}$, Guittin ${ }^{7}$ e Brown e Jones ${ }^{8}$, salientando que os fatores envolvidos são mecânicos, nutricionais, genéticos e infecciosos.

No entanto, Shane e Tully ${ }^{2}$ não concordam que todos os sintomas possam estar presentes em todos os casos.

Huchzermeyer ${ }^{1}$, Shane e Tully ${ }^{2}$, Carbó ${ }^{3}$, Bezuidenhout et $\mathrm{al}^{6}{ }^{6}$ e Deeming ${ }^{9}$ concordam que os sintomas da rotação tibiotársica caracterizam-se pela rotação progressiva lateral do tibiotarso e tarsometatarso, uni ou bilateral, sem distinção de sexo, embora Carbó 3 refira que apesar da rotação de tarsometatarso ser mais frequente, ela também pode estar associada à rotação de falanges, dedos tortos e desvio do tendão gastrocnêmio.

Reconhecemos que alterações de falanges foram menos comuns em nossos achados, o que corrobora com os achados de Carbó ${ }^{3}$.

Huchzermeyer ${ }^{1}$ e Shane e Tully ${ }^{2}$, concordam que problemas com manejo inadequado e deficiências nutricionais, especialmente cálcio e fósforo e vitamina
D3; já Carbó ${ }^{3}$ e Squire e More ${ }^{10}$ dizem que as deformidades de membros são agravadas, quando estas deficiências nutricionais encontram-se associadas.

Huchzermeyer ${ }^{1}$ e Shane e Tully ${ }^{2}$ concordam que problemas com manejo inadequado podem aumentar a incidência de rotação tibiotársica. Vale ressaltar que as propriedades que cederam o material para presente estudo não apresentaram citações inerentes a problemas com manejo.

De acordo com os mesmos autores os filhotes de avestruz são propensos a problemas de pernas, sendo a maioria deles multifatorial, correlacionado com problemas de manejo, já que nesta fase os filhotes ainda não possuem um controle total sobre suas pernas.

Squire e More $^{10}$ e Faquharson e Jefferels ${ }^{11}$ concordam que esta condição tenha sido detectada na fase inicial de crescimento, podendo ser progressiva ou desenvolver-se lentamente, o que concordamos pela progressão dos sinais clínicos relatados nas diferentes propriedades

Embora Bezuidenhout e Burger ${ }^{12}$ tenham apontado uma dominância da rotação tibiotársica do lado direito, observamos uma maior incidência bilateral.

Huchzermeyer ${ }^{1}$, Shane e Tully², Deeming ${ }^{9}$, Bezuidenhout e Burger ${ }^{12}$ e Dick ${ }^{13}$ concordam que a correção em fase inicial pode ser obtida por meio do uso de bandagens ou talas, no entanto, Guittin ${ }^{7}$ afirma que esta condição pode invariavelmente progredir a ponto de uma incapacidade de locomoção, o que apoiamos, pois este procedimento é comum nas propriedades, e quando realizado precocemente tende a diminuir a incidência de rotação tibiotársica, sendo assim sugerimos que tal procedimento seja associado à correção do piso.

Gandini, Burroughs e Bedes ${ }^{14}$ afirmam que a nutrição é um importante aspecto na criação de avestruzes, sugerindo que alta mortalidade e baixa taxa de crescimento em filhotes de avestruzes de 60 dias possa ter origem nutricional, sendo que Brown e 
Jones $^{8}$, Bezuidenhout e Burger ${ }^{12}$, Angel, Sheideler e Sell ${ }^{15}$, Flieg ${ }^{16}$, Shivaprasad ${ }^{17}$ e Stewart ${ }^{18}$ incluem a nutrição como um dos fatores possíveis envolvidos na rotação tibiotársica.

O estudo radiográfico dos ossos e articulações foi apontado por Eziliano ${ }^{19}$, Provasi $^{20}$ e Tycer $^{21}$, como instrumento valioso no diagnóstico de deformidades osteoarticulares, claudicações, deformidades ósseas metabólicas, confirmações de lesões, auxiliando o clínico do diagnóstico, prognóstico, terapêutica, avaliações de infecções, sendo imprescindível o conhecimento da anatomia, radiologia, para uma interpretação correta.

A artrografia permite a observação de estruturas intra-articulares invisíveis ao estudo radiográfico simples, porém este procedimento não teve resultados satisfatórios nos animais mortos e congelados, suspeitando-se que o meio de contraste pode ter tido insuficiente preenchimento, por alteração post mortem do líquido sinovial.

Especial atenção deve-se se dar aos métodos de diagnóstico não invasivos e que consigam de maneira bastante precoce diagnosticar as alterações ósseas em sua fase inicial, procurando oferecer melhor prognóstico, salientando a importância da profilaxia.

Neste experimento trabalhamos apenas com as alterações concernentes a tíbia, tibiotarso, tarsometatarso e falanges, onde identificamos a presença de rotação em tíbia e desvios em falanges.

Em relação ao crescimento longitudinal do osso, ocorre através da proliferação dos condrócitos, produção da matriz óssea, calcificação, hipertrofia e invasão do condrócito hipertrofiado na lacuna ${ }^{22,23,24,25}$.

Pizauro $^{26}$, sugeriu em seu relato que um atraso na apoptose dos condrócitos pode resultar em células hipertrofiadas na placa de crescimento e que a maturação dos condrócitos pode estar associada ao desenvolvimento de lesões ósseas.

Quando associado à radiologia, a microscopia, foi um importante método de diagnóstico, sendo possí- vel de maneira simples, detectar alterações microscópicas, com poucas amostras, podendo desta forma estabelecer um diagnóstico precoce.

Os cortes histológicos não evidenciaram alterações significantes, concernentes à matriz óssea, porém uma persistência de condrócitos nos animais estudados sugeriu a presença de um osso imaturo, o que nos chamou atenção por se tratar de animais adultos.

Vale ressaltar que a persistência de condrócitos nos resultados morfológicos encontrada em animais adultos possa ser indicativa de um fator causal da rotação tibiotársica, suspeitando-se de que estes animais adquirem o peso de um animal adulto, permanecendo a estrutura celular de um animal jovem, devendo o estudo morfológico ser realizado com o propósito de um aprofundamento da pesquisa realizada.

\section{Conclusões}

Os resultados obtidos no desenvolvimento deste estudo, pela análise de 30 avestruzes com lesões sugestivas de rotação tibiotársica ao exame radiográfico simples, avaliadas por meio da artrografia, descrição anatômica e morfológica, permitem-nos concluir que: a maioria das estruturas esqueléticas em avestruzes é semelhante às das demais espécies aviárias e mamíferos, entretanto, a ausência de patela e desvio de tendão gastrocnêmio são fisiológicos, e presentes apenas nesta espécie. A radiografia é um procedimento prático, não invasivo, aplicável no diagnóstico de doenças esqueléticas, tornando possível se obter o máximo de informações a respeito do tempo e extensão da lesão, sendo imprescindível o conhecimento da anatomia para uma correta interpretação. As mudanças radiográficas básicas nas aves, similares àquelas detectadas em mamíferos, e o atraso na apoptose dos condrócitos resultaram em acúmulo de células hipertrofiadas na placa de crescimento, sendo que a maturação dos condrócitos poderá contribuir para a compreensão dos mecanismos responsáveis por esta lesão óssea. 


\section{Agradecimentos}

À Fapesp (Fundação de Amparo à Pesquisa do Estado de São Paulo), pelo financiamento do experimento através do Auxílio Pesquisa processo n. 03-05633/0; à empresa “Brasil Ostrich.", em nome do Prof. Dr. Celso

\section{Referências}

1.HUCHZERMEYER, F. W. Doenças dos avestruzes e outras ratitas. Jabuticabal: Funep, 2000. p. 161-170.

2.SHANE, S. M.; TULLY, T. N. Ratite management, medicine, and surgery. Florida, Malabar: Krieger Publishing Company, 1996. p. 127-146.

3.CARBÓ, C. B. Producción del avestruz: aspectos claves. Madrid: Mundi Pressa, 2003. p. 7-30.

4.CARRER, C.; KORNFELD, M. E. A criação de avestruzes. Pirassununga: C. C. Carrer, 1999. p. 303.

5.SPEER, B. L. An introduction to ratite birds for the avian techinican. In: ANNUAL CONFERENCE OF THE ASSOCIATION OF AVIAN VETERINARIANS, 1991, Nashville. Proceedings... Chicago: Association of Avian Veterinarians, 1991. p. 327-337.

6.BEZUIDENHOUT, A. J.; BURGER, W. P.; REYERS, F.; SOLEY, J. T. Serum and bone-mineral status of ostriches with tibiotarsal rotation. Journal Veterinary Research, v. 61, n. 3, p. 203-206, 1994.

7.GUITTIN, P. Bow leg syndrome in ratite birds. Anicultura Magazine, v. 92, p. 70-79, 1987.

8.BROWN, C. R.; JONES, G. E. Some blood chemical, electrolyte and mineral values from young ostriches. Journal South Africa Veterinary Association, v. 67, n. 3, p. 111-114, 1996.

9.DEEMING, D. C. Biology, production and health. London: CABI Publishing, 1999. p. 358.

10.SQUIRE, B. T.; MORE, S. J. Factors on farms in eastern Australia associated with the development to tibiotarsal rotation in ostrich chicks. Australian Veterinary Journal, v. 76, n. 2, p. 110-117, 1998.

11.FAQUHARSON, C.; JEFFERELS, D. Chondrocytes and longitudinal bone growth; the development of tibial dischondroplasia. Poutry Science, v. 79, n. 7, p. 994-1004, 1998 .

12.BEZUIDENHOUT, A. J.; BURGER, W. P. The incidence of tibiotarsal Rotation in the Ostrich (Struthio Camelus). Journal Veterinary Research, v. 64, p. 159-161, 1993.

13. DICK, K. J. Equine arthrogrphy. Veterinary Radiology , v. 25, n. 2, p. 93-96, 1984.

14.GANDINI, G. C. M.; BURROUGHS, E.; BEDES, H. Preliminary investigation into the Nutrition of Ostrich Chicks (Struthio camelus) Under Intensive Conditions. Journal of the South African Veterinary Association, v. 591, n. 1, p. 39-42, 1986.

15. ANGEL, C. R.; SHEIDELER, S. E.; SELL, J. E. Ratite nutrition.
C. Carrer e do Prof. Dr. Marcelo Kornfiel, pelo fornecimento dos animais utilizados neste experimento e à UNESP de Araçatuba, em nome do Prof. Dr. Manoel Garcia Neto, pelo fornecimento dos animais utilizados neste experimento.

In: TULLY, T. N.; SHANE, S. M. Ratite management, medicine, and surgery. Florida, Malabar: Krieger Publishing Company, 1996. p. 11-30.

16. FLIEG, G. M. Nutritional problems in young ratites. International Zoological Yearbook, v. 13, p. 158-163, 1973.

17.SHIVAPRASAD, H. L. Neonatal mortality in ostriches: an overview of possible causes. In: ANNUAL CONFERENCE OF THE ASSOCIATION OF AVIAN VETERINARIANS, 1993, Nashville. Proceedings... Lake Worth, FL: Association of Avian Veterinarians, 1993. p. 282-285.

18.STEWART, J. W. Ratites. In: RITCHIE, B. R.; HARRISON, G. J.; HARRISON, L. R. Avian medicine, principles and application. Lake Worth, Flórida: Wingers Publishings, 1994. p. $1284-1326$.

19.EZILIANO, L. E. Estudo comparativo entre os exames radiográficos e artroscópicos da articulação metacarpofalangeana de eqüinos puro sangue inglês, submetidos a treinamentos para corrida. 2001. $85 \mathrm{f}$. Dissertação (Mestrado em Medicina Veterinária) - Faculdade de Medicina Veterinária e Zootecnia, Universidade de São Paulo, São Paulo, 2001.

20.PROVASI, A. Achados radiográficos nos ossos terceira falange, sesamóide distal, terceiro metacarpiano, primeira falange e sesamóide proximal, dos membros anteriores de cavalos de corrida assintomáticos da raça Puro Sangue Inglês. 2001. 83 f. Dissertação (Mestrado em Cirurgia) Faculdade de Medicina Veterinária e Zootecnia, Universidade de São Paulo, São Paulo, 2001.

21.TYCER, R. Técnicas radiográficas na prática veterinária. São Paulo: Roca, 1984. p. 220.

22. ANDERSON, H. C. Molecular biology of matrix vesicles. Clinical Orthopaedics and Related Research, v. 314, p. 266280, 1995.

23.FAQUHARSON, C.; JEFFERELS, D. Chondrocytes and longitudinal bone growth; the development of tibial dischondroplasia. Poutry Science, v. 79, n. 7, p. 994-1004, 1998.

24. GERBER, H. P.; FERRARA, N. Angiogenesis and bone growth. Trends in Cardiovascular Medicine, v. 10, p. 223-238, 2000.

25.PRAUL, C. A.; FORS, B. C.; GAY, C. V.; PINES, M.; LEACH, R. M. Gene expression and tibial dyschondroplasia. Poultry Science, v. 79, p. 1009-1013, 2000.

26. PIZAURO, J. M. Discondroplasia tibial: mecanismos de lesão e controle. Revista Brasileira de Ciência Avícola, v. 4, n. 3, p. 169-185, 2002. 